\title{
A case of multiorgan resection for locally advanced stomach cancer
}

\author{
Tevfik Küçükkartallar', Ebubekir Gündeș¹, Hüseyin Yılmaz², Faruk Aksoy'
}

ABSTRACT Extensive surgical resection may be required in locally advanced gastric cancers, with involvement of other organs. Our purpose was to present a case where vascular reconstruction was performed during multiple organ resections in a patient with locally advanced gastric cancer. An emergent laparotomy was performed on a patient who presented with upper gastrointestinal system hemorrhage; and he was diagnosed with locally advanced gastric cancer. Total gastrectomy, D II dissection, pancreaticoduodenectomy, transverse colectomy and end-to-end anastomosis were performed. Additionally, the superior mesenteric vein was partially excised and re-implanted to the portal vein. Neither recurrence nor distant metastasis was observed during the post-operative follow-up. Adjacent organ resections may be necessary for a curative treatment in locally advanced gastric cancer. In addition, partial resection and reconstruction may be required for extensive vascular invasion.

Key Words: Gastric cancer, resection, vascular repair

'Department of General Surgery, Necmettin Erbakan University Meram Faculty of Medicine, Konya, Turkey

${ }^{2}$ Department of General Surgery, Selçuk University Faculty of Medicine, Konya, Turkey

Address for Correspondence Dr. Tevfik Küçükkartallar Department of General Surgery, Necmettin Erbakan University Meram Faculty of Medicine, Konya, Turkey

Phone.: +903322236540

e-mail:

tevfikkk75@hotmail.com

Received: 15.07.2011

Accepted: 10.11.2011

(C) Copyright 2013 by Turkish Surgical Association

Available online at www.ulusalcerrahidergisi.org

\section{INTRODUCTION}

In recent years, the incidence of gastric cancer and mortality related to this pathology has decreased. Nevertheless, with the increase of malignant proximal gastric lesions throughout the world, it continues to be one of the leading causes of cancer related deaths (1). The goal of surgical treatment in gastric cancer is curative resection. In locally advanced gastric cancer, extensive surgical resection of invaded adjacent organs may be required, despite the possibility of perioperative complications (2). In some patients, infiltration of vascular structures may be encountered. In such cases, extended resection is completed with vascular reconstruction. Our goal is to present a case with advanced stage gastric cancer, who required vascular reconstruction during multiple organ resection.

\section{CASE PRESENTATION}

A 51 year old male patient referred to our clinic with upper gastrointestinal (Gl) bleeding from the emergency department was found to have a $3 \times 5 \mathrm{~cm}$ malignant lesion in the gastric antrum with blood stained appearance. The patient's general condition was moderate, he was conscious, with a blood pressure of $90 / 60 \mathrm{mmHg}$ and pulse rate of 120 per minute. Melena was detected in the rectal examination. The hemoglobin value was $6 \mathrm{mg} / \mathrm{dL}$, and hematocrit was 19 . The patient was given fluid-electrolyte replacement as well as 3 units of blood transfusion. The patient had continuous hematemesis and hemodynamic deterioration, therefore an emergent laparotomy was performed. In abdominal exploration, a T4 tumoral mass was detected in the gastric antrum, that has invaded beyond the serosa, invaded the pancreatic head and caused complete obstruction of the colon. Total gastrectomy, D II dissection, pancreaticoduodenectomy, transverse colectomy and end-to-end anastomosis was performed. A tumor infiltration was found at the superior mesenteric vein (SMV) where it is connected to the portal vein, and approximately $1.5 \mathrm{~cm}$ segment was resected. Then, without using grafts, an end -to-side reimplantation was performed directly to the portal vein with $6 / 0$ polypropylene suture. In the postoperative period, the patient was started on low-molecularweight-heparin, and patency of the SMV and portal vein were verified by serial Doppler ultrasound evaluations. The patient was hemodynamically stable, he developed an intra-abdominal abscess on the $6^{\text {th }}$ postoperative day which was successfully treated by percutaneous drainage and was discharged on the $15^{\text {th }}$ postoperative day. Histopathological examination of the specimen revealed a poorly differentiated gastric adenocarcinoma. Lymph node metastases were detected in 20 out of 24 lymph nodes. The patient underwent six cycles of chemotherapy with Cisplatin + Fluorouracil. During the 13 months postoperative follow-up, local recurrence or distant metastasis was not detected. 


\section{DISCUSSION}

In T4 gastric cancers, invasion of adjacent organs are observed at a rate of $3-27.5 \%(3,4)$. In such cases, resection of multiple organs may be necessary to achieve a curative gastrectomy. As part of multiple organ resections, distal pancreatectomy, splenectomy and transverse colectomy is often performed. In some patients with liver metastases metastasectomy is being applied. The lymph node dissection can be done as either $\mathrm{D}$ II, or D III dissection (2). However, pancreatoduodenectomy is usually not needed. Although rare, patients who underwent pancreaticoduodenectomy together with gastrectomy have been reported in the literature (5-7).

There are three routes of pancreatic invasion in gastric cancer. Pancreatic head invasion might occur with lymphatic spread throughout the hepatoduodenal ligament and pancreaticoduodenectomy might be necessary. However, this surgery has a high mortality. Pancreatic body and tail could be infiltrated in lesions located in the middle section of the stomach, by spread through lymphatics around the celiac and mesenteric vessels. In this type of patients, curative resection can be provided with distal pancreatectomy. In addition to these, direct extension of the tumor can also be seen (2). In our patient, due to direct extension of the tumor, complete obstruction of the transverse colon was found together with invasion of the pancreatic head. The patient who underwent emergent surgery only had gastroscopy and routine blood tests in the pre-operative period, imaging methods like ultrasonography or computed tomography could not be performed. Therefore, the spread of the tumor could not be detected in the preoperative period. Total gastrectomy, D II dissection, pancreaticoduodenectomy, transverse colectomy and end-to-end anastomosis was performed. Carboni et al. (2) reported graft reconstruction of the main hepatic artery due to tumor infiltration in a patient with locally advanced gastric cancer. Similarly, we detected infiltration of the SMV during resection, and performed an end -to-side re-implantation to the portal vein.

\section{CONCLUSION}

Lymph node metastasis, liver metastasis and curative resection are considered as three independent factors for long- term survival in patients with locally advanced gastric cancer with spread to adjacent organs. In order to achieve curative resection, resection of involved adjacent organs may be required. In addition, vascular infiltration could be detected with extended resection. In this case, vascular resection and reconstruction may be needed for radical resection.

Peer-review: Externally peer-reviewed.

Author Contributions: Study concept and design - F.A.; Acquisition of data - E.G.; Analysis and interpretation of data - H.Y; Preparation of the manuscript - T.K.

Acknowledgement: We thank Dr. Ahmet Tekin for his contributions in the preparation of this manuscript.

Conflict of Interest: No conflict of interest was declared by the authors.

Financial Disclosure: The authors declared that this study has received no financial support.

\section{REFERENCES}

1. Carboni F, Lepiane P, Santoro R, Lorusso R, Mancini P, Sperduti I, et al. Extended multiorgan resection for T4 gastric carcinoma: 25year experience. J Surg Oncol 2005; 90: 95-100. [CrossRef]

2. Kobayashi A, Nakagohri T, Konishi M, Inoue K, Takahashi S, Itou M, et al. Aggressive surgical treatment for T4 gastric cancer. J Gastrointest Surg 2004; 8: 464-70. [CrossRef]

3. Shinohara T, Uyama I, Kanaya S, Inaba K, Isogaki J, Horiguchi A, et al. Totally laparoscopic pancreaticoduodenectomy for locally advanced gastric cancer. Langenbecks Arch Surg 2009; 394: 733-7. [CrossRef]

4. Saka M, Mudan SS, Katai H, Sano T, Sasako M, Maruyama K. Pancreaticoduodenectomy for advanced gastric cancer. Gastric Cancer 2005; 8: 1-5. [CrossRef]

5. Nunobe S, Hiki N, Ohyama S, Fukunaga T, Seto Y, Yamaguchi T. Survival benefits of pancreatoduodenectomy for gastric cancer: relationship to the number of lymph node metastasis. Langenbecks Arch Surg 2008; 393: 157-62. [CrossRef]

6. Wang XB, Yang LT, Zhang ZW, Guo JM, Cheng XD. Pancreaticoduodenectomy for advanced gastric cancer with pancreaticoduodenal region involvement. World J Gastroenterol 2008; 14: 3425-9. [CrossRef]

7. Chan WH, Cheow PC, Chung AY, Ong HS, Koong HN, Wong WK. Pancreaticoduodenectomy for locally advanced stomach cancer: preliminary results. ANZ J Surg 2008; 78: 767-70. [CrossRef] 